\title{
Comparative Analysis of Hydrodynamics Behavior of Microalgae Suspension Flow in Circular, Square and Hexagonal Shape Photo Bioreactors
}

\author{
Mushfique Shahriar1, Mohammad Iftekhar Monir', Ujjwal Kumar Deb ${ }^{3 *}$ \\ ${ }^{1}$ Department of Mechanical Engineering, Chittagong University of Engineering \& Technology, Chittagong, Bangladesh \\ ${ }^{2}$ Department of Mathematics, Premier University, Chittagong, Bangladesh \\ ${ }^{3}$ Department of Mathematics, Chittagong University of Engineering \& Technology, Chittagong, Bangladesh \\ Email: tanim0907@gmail.com, monir1175@yahoo.com, *ukdeb03@gmail.com
}

How to cite this paper: Shahriar, M., Monir, M.I. and Deb, U.K. (2016) Comparative Analysis of Hydrodynamics Behavior of Microalgae Suspension Flow in Circular, Square and Hexagonal Shape Photo Bioreactors. American Journal of Computational Mathematics, 6, 320-335.

http://dx.doi.org/10.4236/ajcm.2016.64033

Received: October 17, 2016

Accepted: December 18, 2016

Published: December 21, 2016

Copyright $\odot 2016$ by authors and Scientific Research Publishing Inc. This work is licensed under the Creative Commons Attribution International License (CC BY 4.0).

http://creativecommons.org/licenses/by/4.0/

\begin{abstract}
Microalgae based biofuel is an emerging natural source of energy alternative to the fossil fuel. As microalgae are photosynthetic microorganisms, light is one of the limiting factors for its culture. Though many researches have been carried out for findings behind suitable culture system for the proper growth of microalgae, those are confined only to tubular Photo-bioreactor (PBR). This paper aims to make comparison among the horizontal loop photo bioreactors with different cross sections based on the analysis of hydrodynamics behavior. Three different geometrical shapes having vertical cross sections of circular, square and hexagonal PBR, have been proposed taking into account light intensity for microalgae culture. In this study, we simulate the flow dynamics of three types of PBRs and discuss the velocity, pressure and shear stress properties as microalgae endurance capacity depends on them. For the dimension of the three PBRs we considered here, each of them have radius of about $0.05 \mathrm{~m}$ while the length together with bending portion is approximately $20.5 \mathrm{~m}$ for a single loop. From the study, the hydrodynamic behaviors are observed to be quite dissimilar in case of three PBR's. In the straight portion the velocity profile is quite parabolic in tubular but distorted minimally in case of square and hexagonal PBRs. In the middle of the U-loop, a haphazard fluid distribution is noticed. The velocity magnitude and agitation of microalgae cells are higher in hexagonal than in square and tubular. The shear rate is less in case of tubular compared to square and hexagonal. A linear pressure drop is found from the inlet to the outlet for three PBR's. From this comparison, it can be said that the tubular one would be the best option for microalgae culture in case of industrial purposes.
\end{abstract}

\section{Keywords}

CFD, Microalgae, Biofuel, Photo Bioreactor, Simulation 


\section{Introduction}

Sustainable development and efficient use of energy goes on hand which results in environment pollution minimisation and better socio-economic conditions. The Green energy is now a prime talk all around the globe to ensure zero toxic gas emission as global warming and other hazardous pollution caused by burning of fossil fuels are taking the world to an unstable condition. Also increasing consumption of petro-fuel with rapid growth of transportation and population compared to total deposited amount is leading it on the verge of gradual extinction as it is a depleted source of energy [1] [2]. So in this context, oil extracted from organic matter (biofuel) has attracted the scientist and researchers as it is eco-friendly and sustainable. Biofuel has a great potential to mitigate the continuous demand in every aspects. The mission of negative carbon emission would be fulfilled to a great extent by large scale production of biofuel. Biofuel production may become much more economical than fossil fuel. Extensive research has been carried out for the last five decades regarding the development of the biofuel technology. The developed countries of the world have emphasized and investing money to increase the productivity level of biofuel. So it can be foreseen to be the fuel of future [3] [4] [5].

Biofuel is referred to as solid, liquid or gaseous compound obtained from organic matter. The main advantage of it over fossil fuel is that it is non-toxic and biodegradable [2]. Biofuel production is categorized into three generations. The first generation biofuel such as soybean, rapeseed, sunflower, palm oil has some environmental limitations as they compete with cultivable land for food production. The second generation biofuel such as agricultural residues, wood residues, non-edible oil or sugarcane has issues of environmental hazard [6] [7]. So to overcome those drawbacks microalgae based biofuel has been appeared as a potential source of biodiesel which ensures the clean energy for better environment.

Microalgae are eukaryotic and prokaryotic photosynthetic microorganisms. They convert water and $\mathrm{CO}_{2}$ into sugar i.e. lipid and carbohydrate in photosynthesis process by means of sunlight. The sugar contents are subsequently used to extract oil. In the very beginning, microalgae have harvested for some pharmaceutical purposes, waste water treatment, cosmetics and poultry food. As the time passing by, it has got importance in fuel sector due to its almost double productivity level of biomass and it grows 100 times faster than any terrestrial plants. So the challenging task for the researchers is to develop congenial culture system for high productivity of microalgae [8] [9] [10] [11].

Microalgae culture system requires supply of light, $\mathrm{CO}_{2}$, nutrients but availability of light is the first and foremost concern. Adequate exposure to light of every cell in the culture system ensures the uniform growth of microalgae, yet it is a challenging task due to proper design consideration of culture system. Generally, two types of culture systems are available such as traditional open pond system and closed photo bioreactor system. The open pond includes shallow big ponds, circular ponds, tanks and raceway pond but raceway pond has been commonly used for culture [12] [13]. The raceway pond method facilitates economic and easier process of culture but has a lot of disadvantages include 
poor stirring mechanism so mass transfer rate is very low. Hence less mass productivity, insufficient utilization of sunlight close to the bottom surface of pond and contamination risk due to fast growing heterotrophs and predators have hindered the commercial production. On the contrary, the closed photo bioreactor technology is free from those drawbacks to a great extent. But, photobioreactor which is promising to culture of microalgae is still under consideration as better geometric shape and proper growth model are associated with it [14]. Maximum sunlight capturing capacity and minimum space requirement are first and foremost condition in designing a photo bioreactor. Both the outdoor and the indoor cultivation process are in vogue but for large scale production outdoor cultivation is required as to utilize natural source of light. The photo bioreactors such as bubble column, torus, helical and stirred tank have low illuminated surface area. But for outdoor cultivation large illuminated surface area is needed. In this context, U-loop horizontal tubular photo bioreactor is more efficient than other considering their large illumination area and easily scaling up capacity [15] [16] [17] [18]. From this point of view, investigations were limited only to analyse the growth model in case of horizontal u-loop tubular photo bioreactor. In this work we have conducted CFD comparison analysis among different shape horizontal photo bioreactors as simulation plays a vital role to predict the result before going to experiment on a real test bed. We hereby proposed vertically square and hexagonal shape horizontal PBRs besides tubular PBR. As hydrodynamics, light regime, shear stress and pressure distribution are most important parameters to decide which one is the best suited to microalgae growth, so we consider these parameters for result analysis.

In our simulation only the microalgae suspension is considered to obtain fully developed single phase laminar flow with no slip condition at the wall. Here, we have focused on the comparison of fluid behaviour in U-loop portion other than straight part as shear rate and rate of cell damage is high there.

The rest of the paper is organized as follows. The theoretical framework is described in the Section 2; Section 3 describes the methodology of the mathematical model development; results and conclusions are presented in the Section 4 and the Section 5 respectively.

\section{Theoretical Framework}

\subsection{Creeping Flow}

The creeping flow model hereby used in our simulation to explain the flow behaviour is a branch of single phase flow and works with fluid flow having very low Reynolds number. In case of creeping flow the inertia term of the Navier-Stokes equation has been neglected. As it occurs in fluid systems with high viscosity and micro scale geometry, so it can be used to analyse the flow behaviour of microalgae.

The single phase creeping flow model follows the Navier-Stokes equation which is in general form as follows:

$$
\nabla \cdot \boldsymbol{u}=0
$$




$$
\frac{\partial \boldsymbol{u}}{\partial t}+\rho(\boldsymbol{u} \cdot \nabla) \boldsymbol{u}=\nabla \cdot\left[-P \boldsymbol{I}+\eta\left(\nabla \boldsymbol{u}+\nabla \mathbf{u}^{\mathrm{T}}\right)\right]+\boldsymbol{F}
$$

where $\rho$ is the density; $\boldsymbol{u}$ is the velocity vector; $p$ is the pressure; $\boldsymbol{\tau}$ is the viscous stress tensor and $F$ is the body force vector.

\subsection{Shear Stress}

In case of all three kinds of PBR, hydrodynamic forces will obviously cause high shear stress which affects the culture of microalgae by causing constraint to its flow and growth which results in huge cell damage [5]. Shear stress will also determine which PBR is conforming to microalgae culture. The shear stress is determined by the following equation.

$$
\tau=\frac{\partial \boldsymbol{u}}{\partial y}
$$

where $\tau, \boldsymbol{u}, y$ denote shear stress, velocity of flow and direction normal to the flow respectively.

\section{Methodology}

As in this paper, our focus is on the comparative analysis of flow phenomenon for three PBRs, thus we conduct the study in two steps: Mathematical model development and numerical simulation.

\subsection{Mathematical Model}

\subsubsection{Geometry}

Each of the three profiles has same hydraulic diameter, length and radius of the curvature. The radius is $0.025 \mathrm{~m}$, length is approximately $20.4 \mathrm{~m}$ and radius of curvature at U-loop portion is $0.4 \mathrm{~m}$. As all the parameters involved with geometric construction are constant so we can build up a common sketch for tubular, square and hexagonal shape PBR which is shown in Figure 1. In case of the tubular PBR the hydraulic diameter is equal to the diameter of profile. For calculating the hydraulic diameter for square and hexagonal shape the following equation is used

$$
D_{h}=\frac{4 A}{S}
$$

where $D_{h}$ is the hydraulic diameter; $A$ is the area of cross section; $s$ is the wetted perimeter.

\subsubsection{Computational Domain Development}

For computation every domain is placed along $x-y$ plane horizontally. $Z$ axis is perpendicular to flow direction. Table 1 shows the faces, edges and intersecting points for the three PBR's.

The surface area and working volume are shown in Table 2.

The domains of three PBR's are presented in Figure 2. 


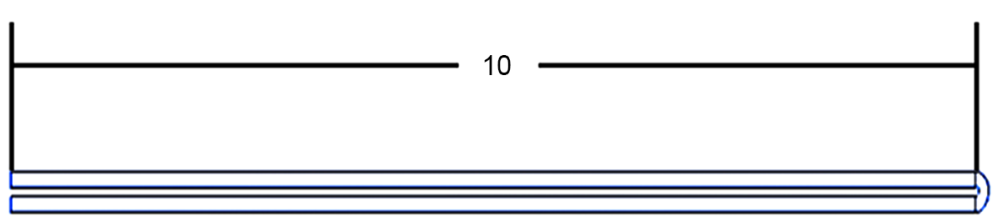

(a)

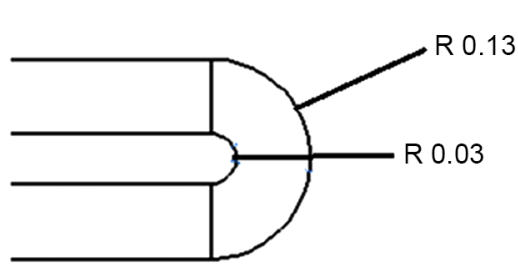

(b)

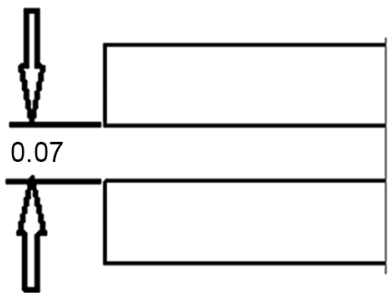

(c)

Figure 1. (a) Dimension of length of straight portion of the PBR (tubular, square, hexagonal); (b) Inner and outer radius of the curvature; (c) Space between straight portions.

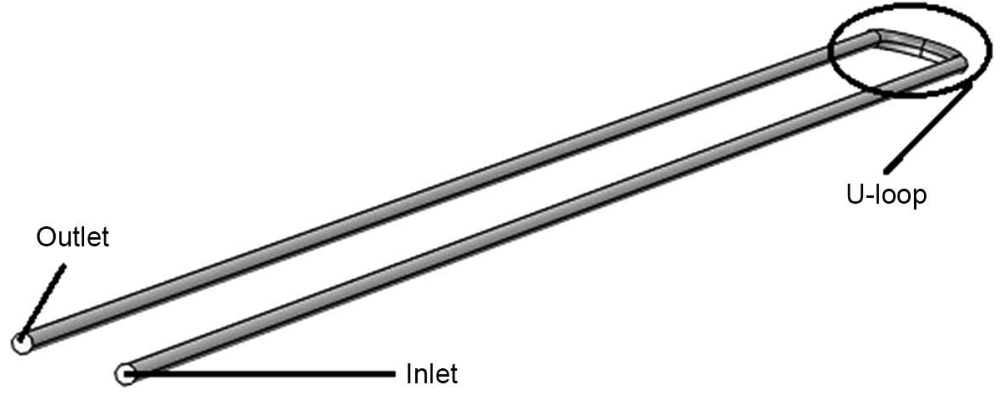

(a)

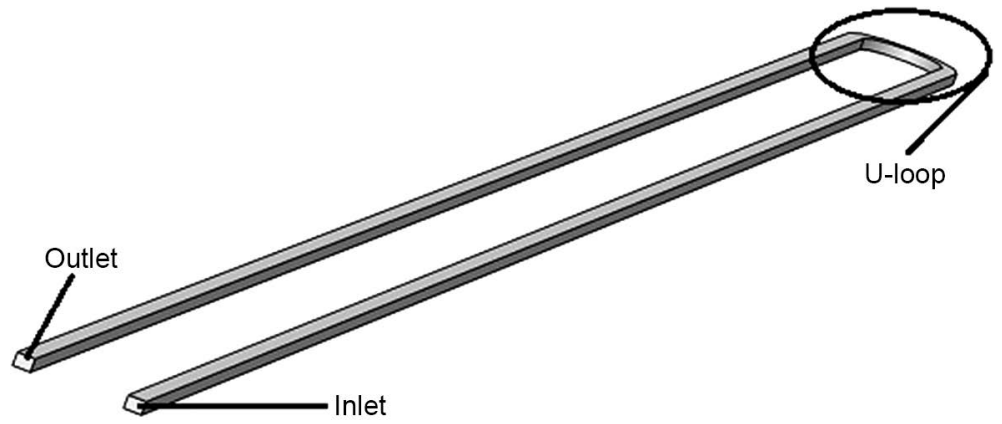

(b)

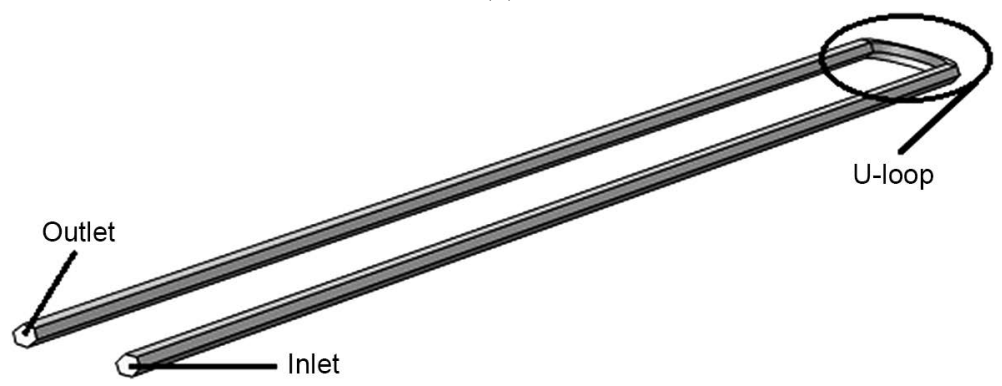

(c)

Figure 2. (a) Tubular PBR showing inlet, outlet and U-loop; (b) Square PBR showing inlet, outlet and U-loop; (c) Hexagonal PBR showing inlet, outlet and U-loop. 
Table 1. Faces, edges and points of the PBR's.

\begin{tabular}{cccc}
\hline PBR's shape type & Faces & Edges & Points \\
\hline Tubular & 8 & 18 & 12 \\
Square & 10 & 24 & 16 \\
Hexagonal & 20 & 42 & 24 \\
\hline
\end{tabular}

Table 2. Surface area \& working volume of PBR's.

\begin{tabular}{ccc}
\hline PBR's shape type & Surface area $\left(\mathrm{m}^{2}\right)$ & Working volume $\left(\mathrm{m}^{3}\right)$ \\
\hline Tubular & 6.351 & 0.1567 \\
Square & 8.124 & 0.2026 \\
Hexagonal & 7.036 & 0.1755 \\
\hline
\end{tabular}

\subsubsection{Governing Equations}

As temperature variation is low and the density is constant, microalgae suspension is considered to be incompressible fluid so Equation (1) reduces to

$$
\nabla \cdot \boldsymbol{u}=0
$$

and Equation (2) becomes

$$
\rho \frac{\partial \mathbf{u}}{\partial t}+\rho(\boldsymbol{u} \cdot \nabla)=\nabla \cdot \sigma+\rho g
$$

where $\sigma$ is the stress tensor and $g$ is the gravity, $\sigma$ can be expressed as

$$
\sigma=-\rho I+2 \eta D(v)
$$

where, $\eta=$ viscosity of the fluid; $D(v)=$ rate of deformation. The viscosity $\eta(t)$ in Equation (7) is determined by

$$
\eta(t)=\eta_{0} \eta_{r}(t)
$$

The relative viscosity $\eta_{r}(t)$ relating to the concentration is then used and determined by

$$
\eta_{r}(t)=1+\varepsilon C(t)
$$

where $\varepsilon$ is the Einstein's coefficient [19]. Based on the experiment conducted by HonNami and Kunito [20], the concentration function $C(t)$ in Equation (9) is given by the logistic Equation (10)

$$
C(t)=C_{0}+\frac{A}{1+B \mathrm{e}^{-\mu t}}
$$

where $\mu$ is the constant growth rate of microalgae cells; $C_{0}$ is the initial concentration of the suspension and $A$ and $B$ are constants.

\subsubsection{Boundary and Initial Conditions}

For simulation we considered the no slip condition on the wall of the tube and the zero 
normal stress at the outlet for all the three domains, as follows:

$$
\begin{gathered}
\boldsymbol{u}=0 \\
{\left[-P I+\eta(t)\left(\nabla \boldsymbol{u}+(\nabla \boldsymbol{u})^{\mathrm{T}}\right)\right] n=0}
\end{gathered}
$$

\subsection{Mesh Design}

\subsubsection{Mesh Generation}

To implement the Navier-Stokes equation with incompressible flow in microalgae suspension, mesh generation is required for calculation. In our study we use normal mesh. Table 3 shows the mesh elements, minimum and average quality for three PBR's.

The numbers of vertex, edge and boundary elements are given in Table 4.

Figure 3 (a) shows the mesh design of the U-loop of tubular, square and hexagonal shape PBR's and Figure 3(b) shows the vertical cross sectional views of the corresponding PBR's.

\subsubsection{Grid Sensitivity Analysis}

A grid sensitivity test is performed in case of tubular photo bioreactor. Both normal and coarse mesh is formed to make comparative study of mesh quality for better result in case of time dependent study. Table 5 shows the comparison of elements between normal and coarse mesh.

From Table 5 we observed that mesh quality and elements both are better in normal mesh.

\subsection{Simulation Parameters}

The main goal of our study is to acquire in depth knowledge of flow behaviour for three different shape PBR's. For our simulation we use the COMSOL Multiphysics version 4.2a package. The parameters that are used as input data are given in Table 6.

Table 3. Total elements \& quality of PBR's.

\begin{tabular}{cccc}
\hline PBR's shape type & Total elements & Minimum quality & Average quality \\
\hline Tubular & 153,356 & 0.03063 & 0.6759 \\
Square & 194,771 & 0.07511 & 0.6691 \\
Hexagonal & 153,122 & 0.09728 & 0.6467 \\
\hline
\end{tabular}

Table 4. Mesh parameters of the PBR's.

\begin{tabular}{cccc}
\hline PBR's shape type & Vertex elements & Edge elements & Boundary elements \\
\hline Tubular & 12 & 1825 & 28,472 \\
Square & 16 & 2416 & 19,158 \\
Hexagonal & 24 & 5145 & 30,746
\end{tabular}


Table 5. Comparison between normal and coarse mesh.

\begin{tabular}{ccccc}
\hline Mesh Type & Total elements & Minimum quality & Average quality & Mesh volume $\left(\mathrm{m}^{3}\right)$ \\
\hline Normal & 153,526 & 0.1506 & 0.6948 & 0.1551 \\
Coarse & 53,189 & 0.06733 & 0.5986 & 0.1519 \\
\hline
\end{tabular}

Table 6. Parameters used for simulation.

\begin{tabular}{ccc}
\hline Name & Value & Description \\
\hline$g$ & $9.8 \mathrm{~m} / \mathrm{s}^{\wedge} 2$ & Gravity acceleration \\
$\eta_{0}$ & $0.001\left[\mathrm{~Pa}^{\star} \mathrm{s}\right]$ & Water viscosity \\
$C_{0}$ & 0.55 & Constant parameter \\
$B$ & 200 & Constant parameter \\
$A$ & 1 & Constant parameter \\
$\mu$ & $0.063[1 / \mathrm{h}]$ & Maximum growth rate \\
\hline
\end{tabular}

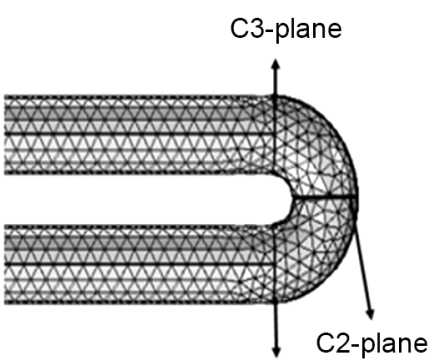

C1-plane

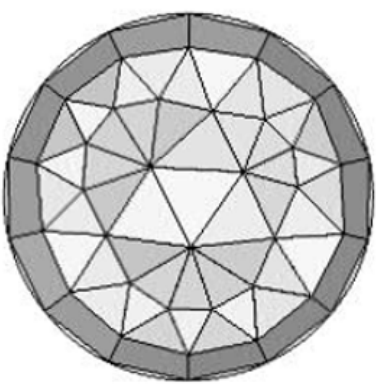

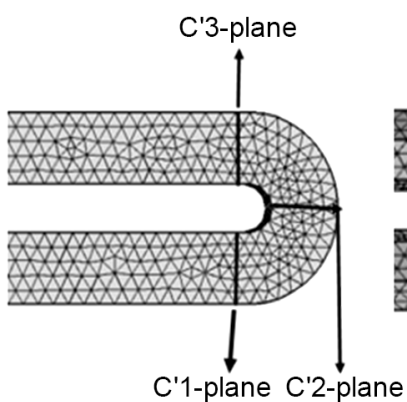

(a)

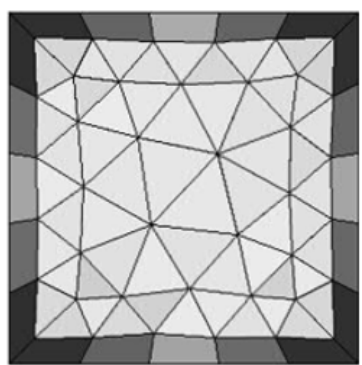

(b)

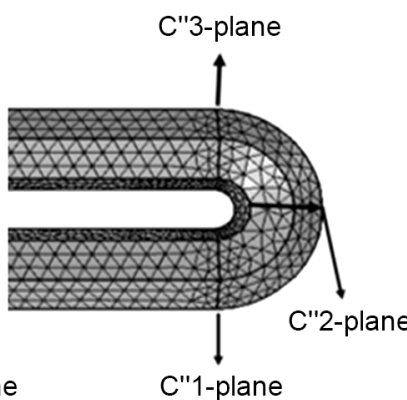

C"1-plane

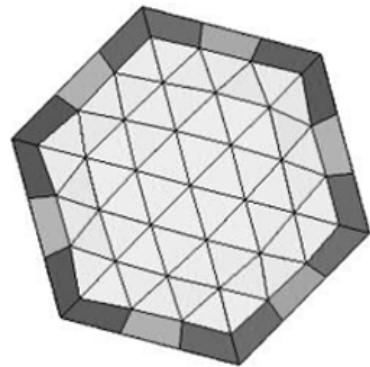

Figure 3. (a) Mesh design for three PBR's showing planes C1, C2, C3 (C1: Entrance of U-loop, C2: Middle of U-loop, C3: Exit of U-loop); (b) Cross section of normal mesh for circular, square \& hexagonal PBR's respectively.

\section{Results and Discussion}

During the simulation, the solver was configured as time dependent. For achieving better and comparative results, we ran the simulation for the seventh day of microalgae culture as microalgae growth can clearly be observed from this day. The time range was $(540,000,10,540,050)$ seconds. From those analyses significant changes are noticed for 
three PBR's. For comparison, we analyse the results for 50 second. The topics of interest in our result analysis include grid independency, velocity distributions, pressure profiles through the ducts curvature and shear stress on the wall of ducts. The shear stress at the middle of the U-loop is the prime concern in this paper as it helps to find out which one is conforming to less cell damage. To identify the fluid behaviour for different profiles the three PBR's are fragmented in different three cross sections.

As mesh size plays a vital role in case of accuracy, so satisfactory computational accuracy can be achieved by continuously changing the meshes until the results from two trials lead to very close to each other [14]. As we previously discussed about the grid independency, some results regarding velocity magnitude are shown in Figure 4 and Figure 5 for normal and coarse mesh in case of tubular PBR.

In Figure 4(a), for coarse mesh the velocity magnitude is $0.9168 \mathrm{~m} / \mathrm{s}$ and in Figure 4(b), for coarse mesh it is $0.9316 \mathrm{~m} / \mathrm{s}$. The both cross sections are taken at the middle of U-loop. From Figure 5(a) the maximum velocity at straight portion is 0.75 for coarse mesh and from Figure 5(b) it is 0.8 for normal mesh.

\subsection{Velocity Profile}

The velocity profile helps to predict the fluid behaviour in the three PBR's. Higher velocity magnitude makes a haphazard distribution of fluid in U-loop in case of all ducts. But the challenging task is to investigate which one has comparatively less velocity magnitude. In Figure 6, the velocity profiles along XY-plane are shown of three PBR's at 50 second respectively.

From Table 7 it is observed that at 50 s lower velocity magnitude is noticed in case of the tubular PBR whereas the square and the hexagonal show almost similar behaviour and higher value than the tubular with inlet velocity $0.5 \mathrm{~m} / \mathrm{s}$.

In Figure 7 the vertical cross sectional views for three PBR's at the straight portion, entrance of U-loop, and middle of U-loop and outlet of U-loop are shown for $50 \mathrm{~s}$ which also exhibits the same phenomenon as it is found along XY-plane. The cross sectional views are taken in $\mathrm{ZX}$ and $\mathrm{YZ}$ planes. From the cross sectional views, it is clear

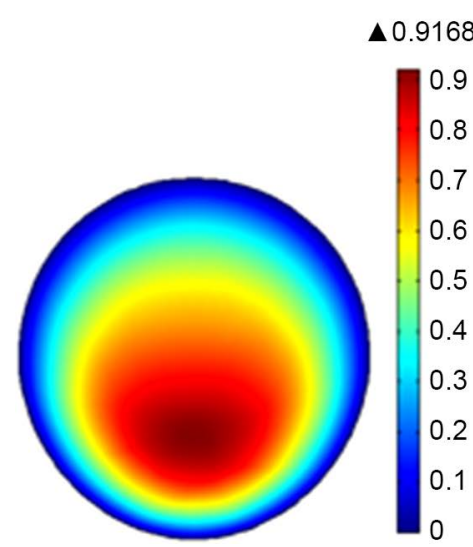

(a)

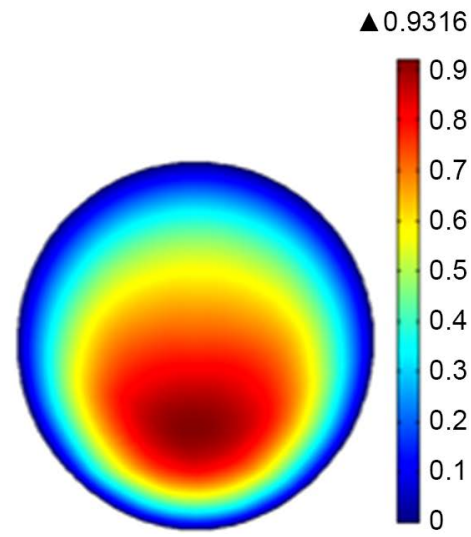

(b)

Figure 4. Velocity at the middle of U-loop of tubular PBR for (a) coarse mesh (b) normal mesh. 


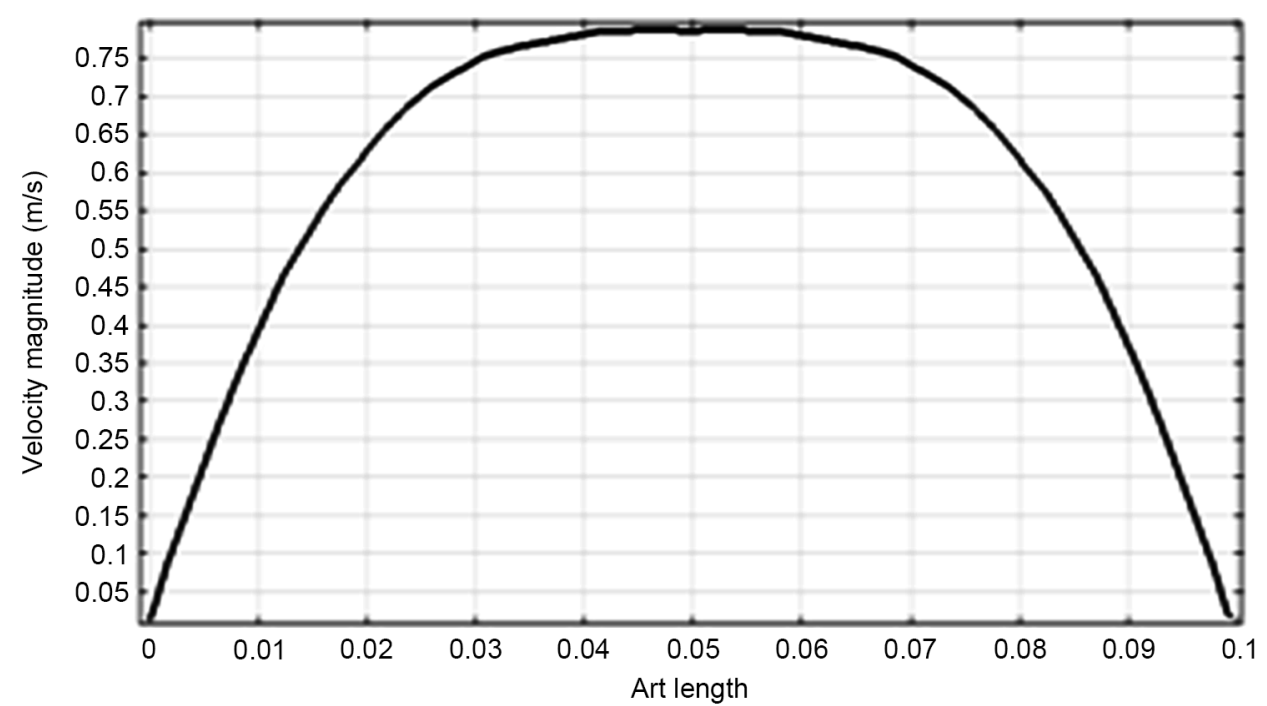

(a)

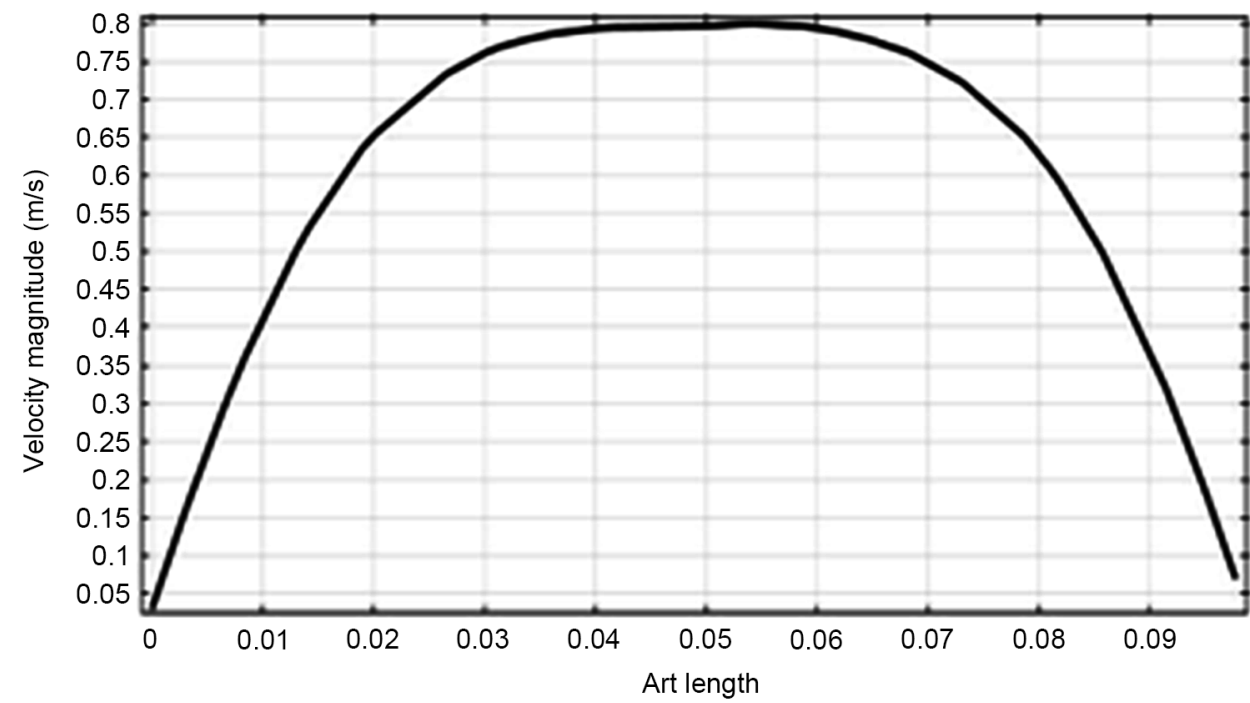

(b)

Figure 5. Velocity profile at straight portion of tubular PBR for (a) coarse mesh (b) normal mesh.

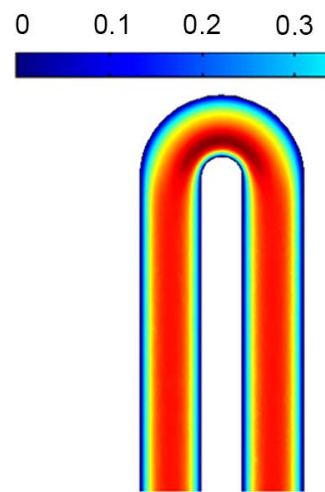

(d)

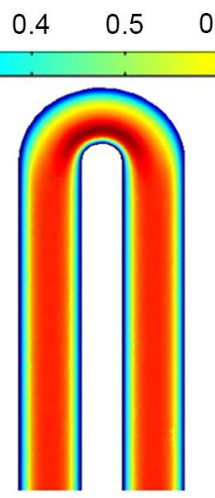

(e) $\begin{array}{lll}0.7 & 0.8 & 0.9\end{array}$

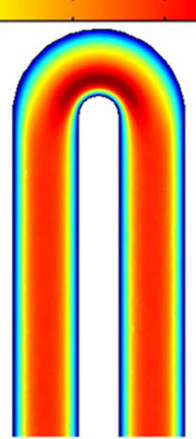

(f)

Figure 6. Velocity profile along XY-plane for (d) tubular (e) square (f) hexagonal ducts at $50 \mathrm{~s}$. 
Table 7. Maximum velocity magnitude at $50 \mathrm{~s}$.

\begin{tabular}{cc}
\hline PBR's shape type & Max. Velocity $(\mathrm{m} / \mathrm{s})$ \\
\hline Tubular & 0.9287 \\
Square & 0.95 \\
Hexagonal & 0.95 \\
\hline
\end{tabular}

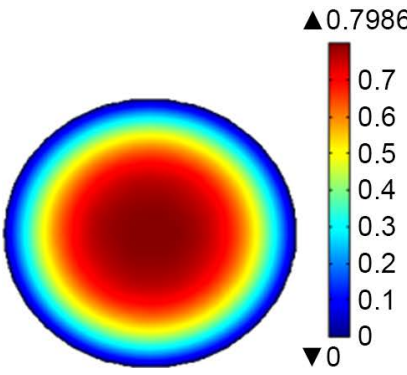

(a)

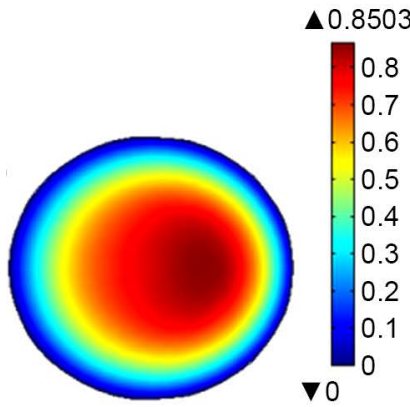

(d)

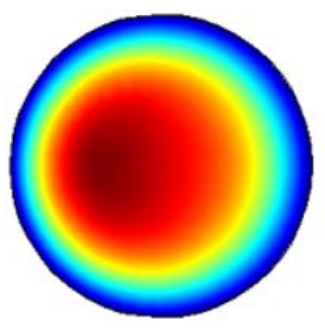

(g)

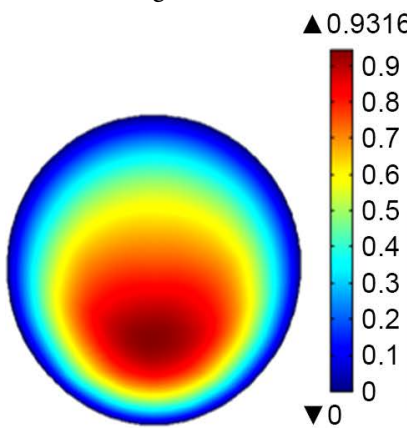

(j)

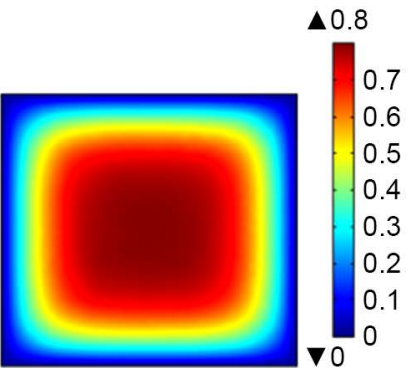

(b)

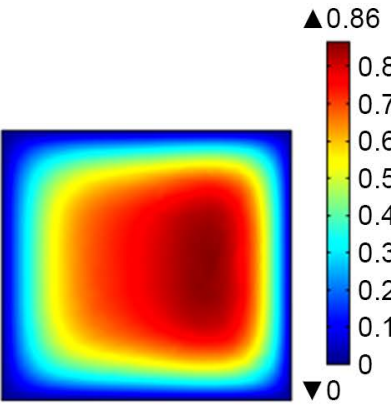

(e)

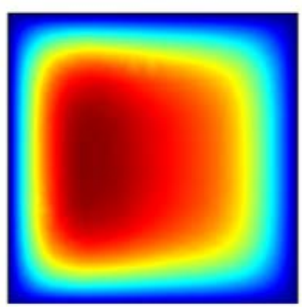

(h)

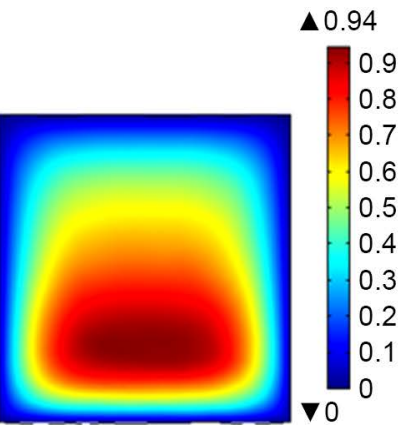

(k)

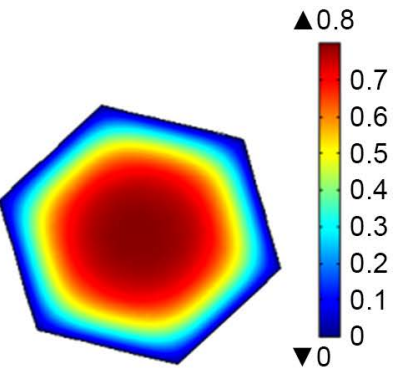

(c)

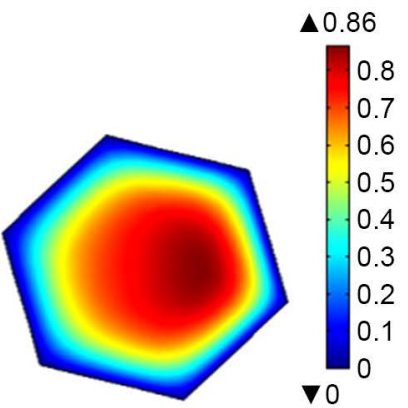

(f)

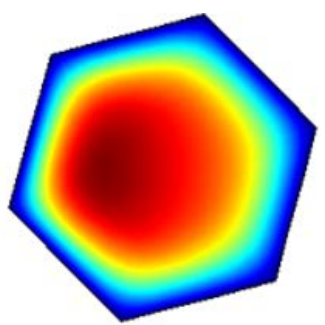

(i)

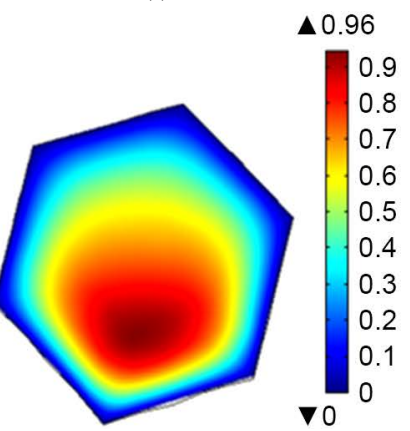

(l)

Figure 7. Velocity profile at arc length $=5 \mathrm{~m}$; (a), (b), (c) at $10 \mathrm{~m}$; (d), (e), (f) at $10.4 \mathrm{~m}$; at middle portion of U-loop respectively at 50s [(a), (d), (b), (e) \& (c), (f) have same legend respectively]. 
that higher agitation and speed of particles region is adjacent to the wall of small radius of curvature for three ducts in U-loop portion. For $50 \mathrm{~s}$ in every case, tubular one shows less speed than square and hexagonal.

The velocity distribution graphs for the tubular, the square and the hexagonal shape PBR are shown in Figure 8. The graphs are taken at time $50 \mathrm{~s}$. The velocity distribution is completely parabolic in the straight portion of every PBR but at the entrance of U-loop, middle of U-loop and outlet of U-loop the shapes are distorted from its regular parabolic shape as the turbulence create in the U-loop portion.

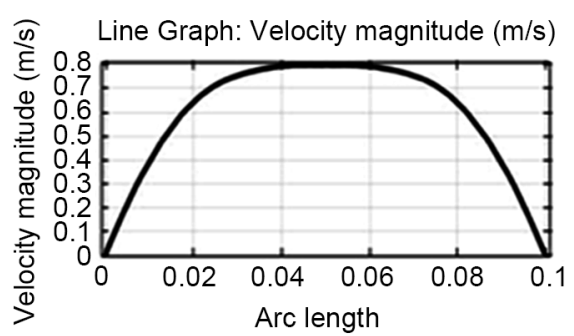

(a)

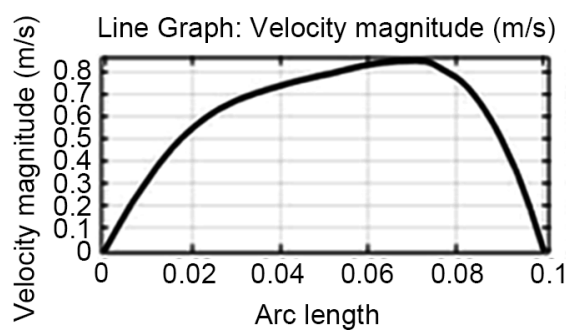

(d)

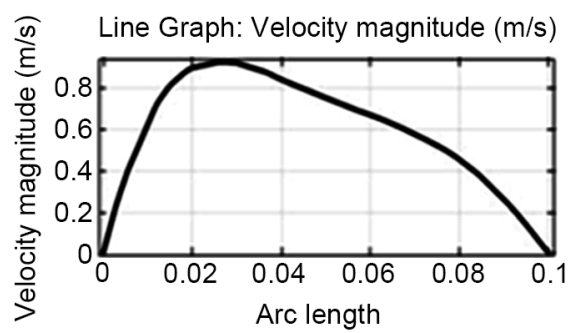

(g)

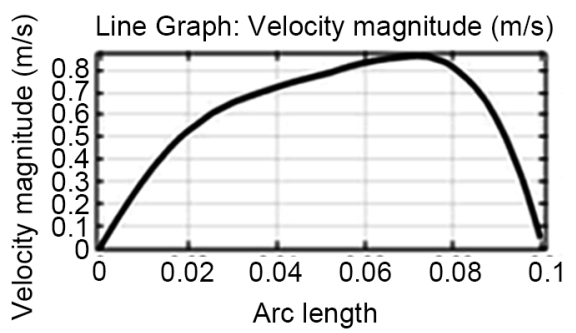

(j)

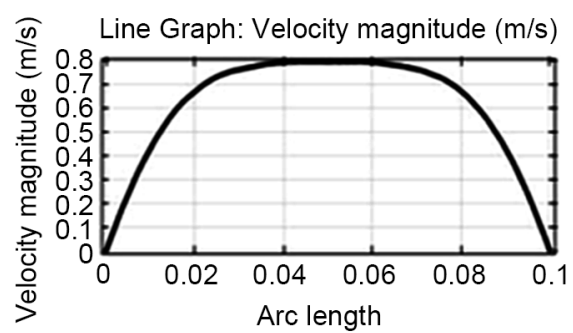

(b)

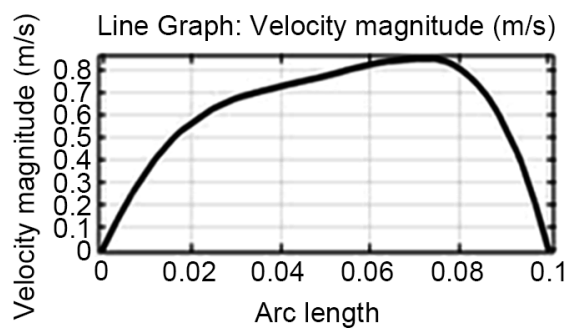

(e)

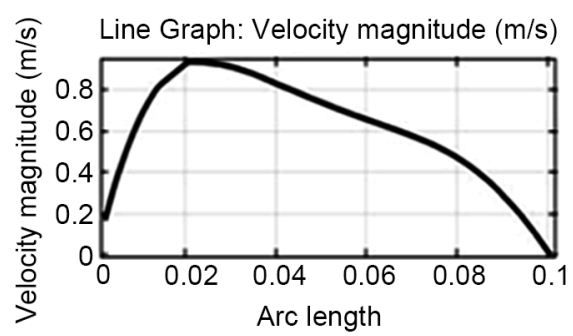

(h)

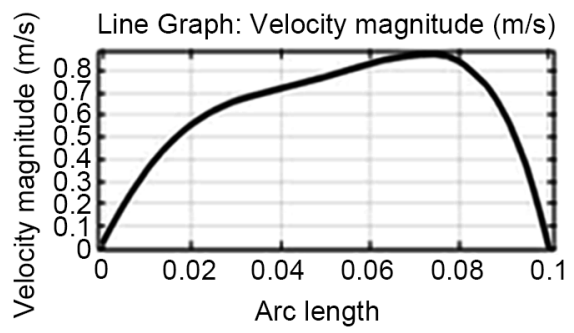

(k)

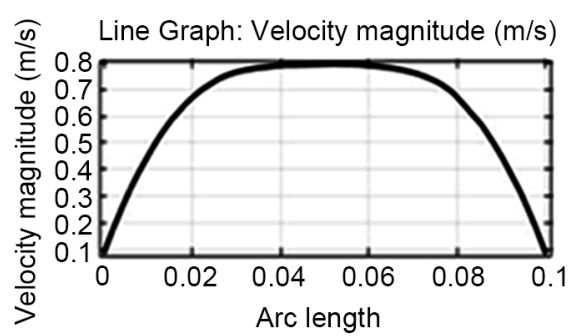

(b)

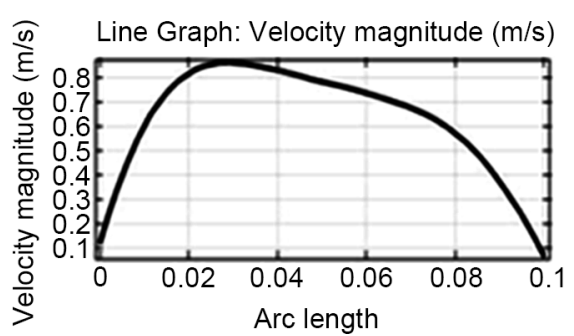

(f)

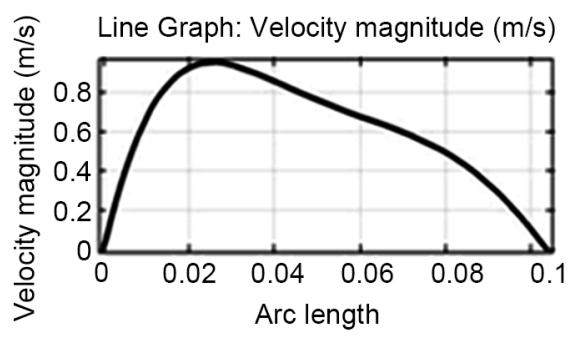

(i)

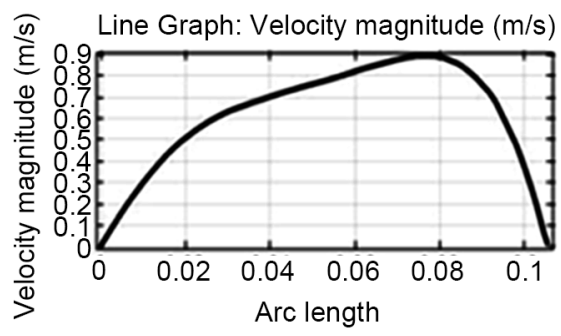

(I)

Figure 8. (a), (b), (c) at arc length = $5 \mathrm{~m}$; (d), (e), (f) at $10 \mathrm{~m}$ (entrance of U-loop); (g), (h), (i) at $10.2 \mathrm{~m}$ (middle portion of U-loop); (j), (k), (l) at $10.4 \mathrm{~m}$ (outlet of U-loop) at $50 \mathrm{~s}$. [In each row the graphs are for tubular, square and hexagonal PBR respectively from left to right]. 


\subsection{Shear Stress}

As the shear stress distribution indicates to predict which one will show better performance to lessen cell damage, we have analysed the shear rate for three different cross sections of U-loop which are presented in Figure 9.

By comparing three different geometrical shape of PBR, less shear stress is observed for the tubular PBR. Table 8 shows the maximum shear stress for different cross sections of three PBR's.

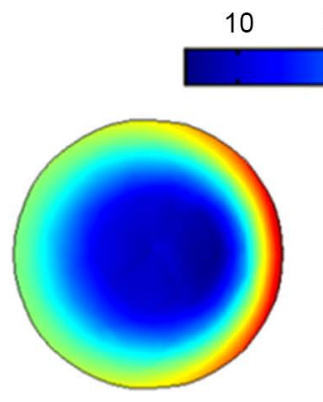

Pc1

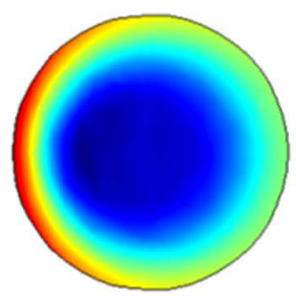

Pc4

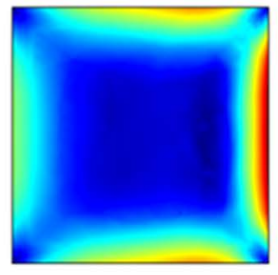

$\mathrm{Pc} 2$

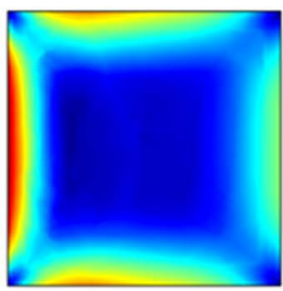

$\operatorname{Pc5}$

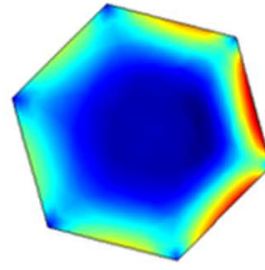

$\mathrm{Pc3}$

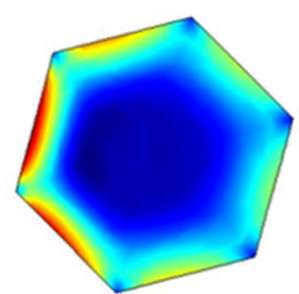

Pc6

$\begin{array}{llllllllll}10 & 20 & 30 & 40 & 50 & 60 & 70 & 80 & 90 & 100\end{array}$

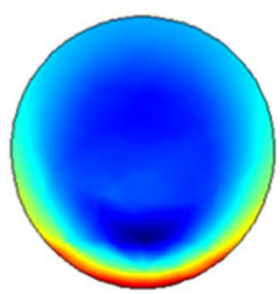

$\mathrm{Pc7}$

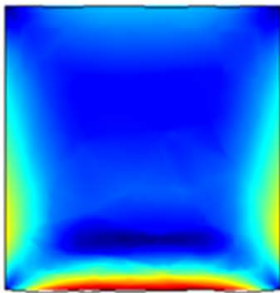

Pc8

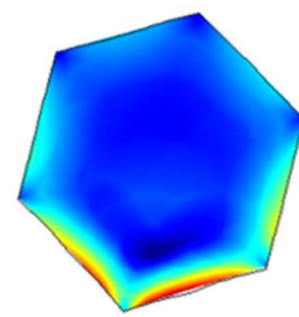

Pc9

Figure 9. Shear stress distribution at three different cross sections: at the inlet of U-loop (Pc1: tubular, Pc2: square, Pc3: Hexagonal), middle of U-loop (Pc4: tubular, Pc5: square, Pc6: Hexagonal), outlet of U-loop (Pc7: tubular, Pc8: square, Pc9: Hexagonal).

Table 8. Minimum shear stress for three PBR's.

\begin{tabular}{cccc}
\hline PBR's shape type & Inlet of U-loop & Middle of U-loop & Outlet of U-loop \\
\hline Tubular & 66.645 & 89.281 & 66.645 \\
Square & 77.2 & 103 & 77.2 \\
Hexagonal & 75.1 & 106 & 75.1
\end{tabular}




\subsection{Pressure Distribution}

The pressure profiles are uniform from inlet to outlet for all three types of geometry. From Figure 10, we can see pressure along the ducts in XY-plane of tubular PBR. Table 9 shows the value of maximum pressures. The pressure is slightly fluctuates at the middle of U-loop for all PBR's which are also observed from line graph shown in Figure 11 .

\section{Conclusion}

In our study, we simulate the flow dynamics of three types of PBR's and discuss the velocity, pressure and shear stress properties as microalgae endurance capacity depends on them. For all the cases, in the U-loop portion, higher velocity exists than any other parts but always the speed is less and moderate in tubular PBR than others. The velocity distribution in tubular PBR is better suited to the culture of microalgae. As shear stress is mostly important factor for microalgae culture, we have analysed our results especially for U-loop portion and less shear stress is found in tubular shape than rest

$\begin{array}{lllllllll}0 & 10 & 20 & 30 & 40 & 50 & 60 & 70 & 80\end{array}$

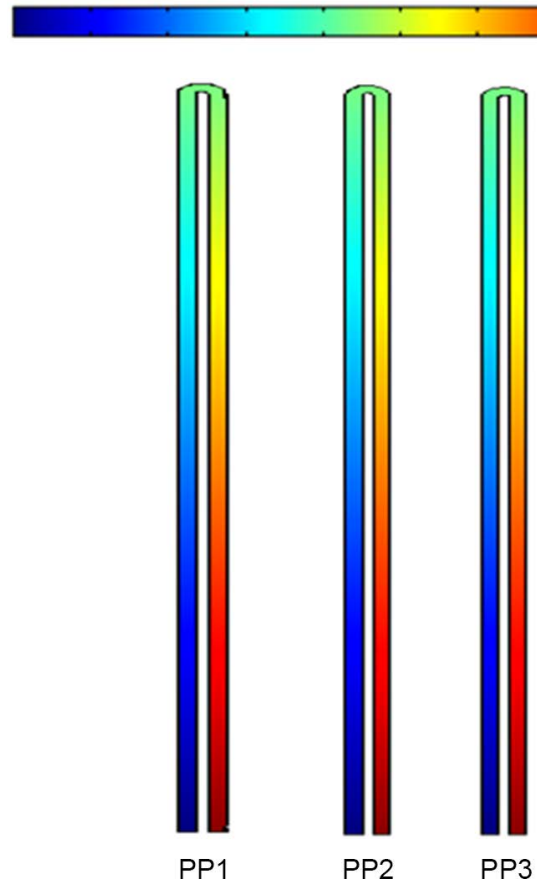

Figure 10. Pressure distribution along the ducts (pp1: Tubular, PP2: Square, PP3: Hexagonal).

Table 9. Maximum pressure along the PBR's.

\begin{tabular}{cc}
\hline Photobioreactor & Maximum pressure \\
\hline Tubular & 83 \\
Square & 87.641 \\
Hexagonal & 84.8
\end{tabular}




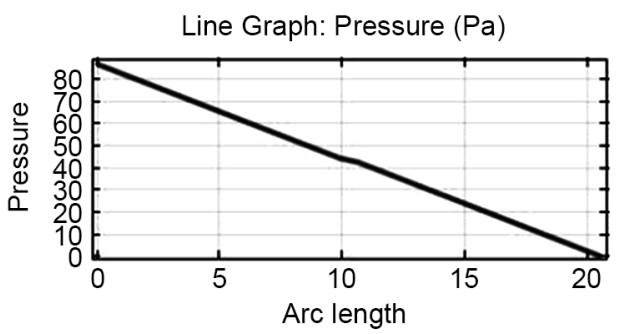

P1

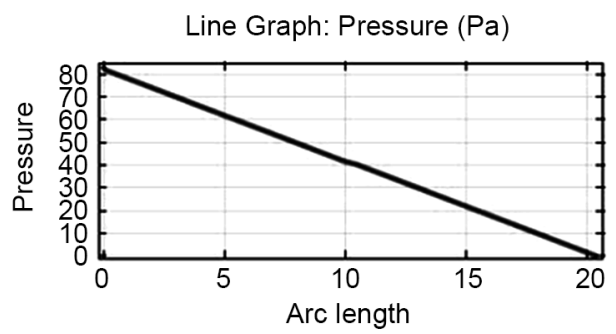

$\mathrm{P} 2$

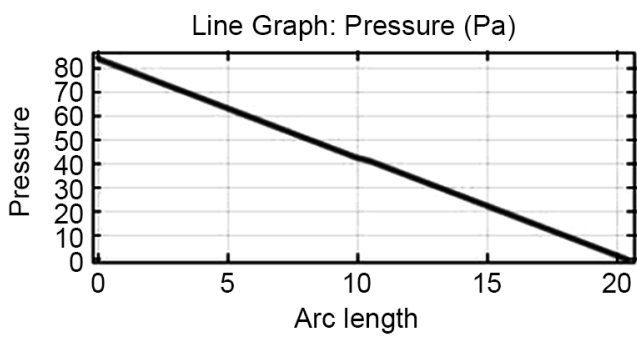

P3

Figure 11. Pressure profile along the PBR's arc length (P1: Tubular, P2: Square, P3: Hexagonal).

of the PBR's. So from our analysis it can be said that tubular PBR is the best choice for microalgae culture. Though pressure profile is always uniformly decreasing from inlet to outlet for all PBR's, a little fluctuation is found in U-loop portion as for haphazard distribution of fluid. But the pressure is always less in tubular PBR. So the simulation result of fluid properties velocity, shear stress and pressure indicates that the tubular one shows better agreement for the culture of microalgae.

\section{Acknowledgements}

The authors are gratefully acknowledged for the technical supports to the Centre of Excellence in Mathematics, Department of Mathematics, Mahidol University, Bangkok10400, Thailand, and the Simulation Lab, Department of Mathematics, Chittagong University of Engineering \& Technology, Chittagong, Bangladesh.

\section{References}

[1] Brennan, L. and Owende, P. (2010) Biofuels from Microalgae-A Review of Technologies for Production, Processing, and Extractions of Biofuels and Co-Products. Renewable and Sustainable Energy Reviews, 14, 557-577. https://doi.org/10.1016/j.rser.2009.10.009

[2] Lam, M.K. and Lee, K.T. (2012) Microalgae Biofuels: A Critical Review of Issues, Problems and the Way Forward. Biotechnology Advances, 30, 673-690.

https://doi.org/10.1016/j.biotechadv.2011.11.008

[3] Gavrilescu, M. and Chisti, Y. (2005) Biotechnology-A Sustainable Alternative for Chemical Industry. Biotechnology Advances, 23, 471-499. https://doi.org/10.1016/j.biotechadv.2005.03.004

[4] Chisti, Y. (2007) Biodiesel from Microalgae. Biotechnology Advances, 25, 294-306. https://doi.org/10.1016/j.biotechadv.2007.02.001

[5] Ramírez-Duque, J.L. and Ramos-Lucumi, M.A. (2011) Hydrodynamic Computational Evaluation in Solar Tubular Photobioreactors Bends with Different Cross Sections. CT\&F: 
Ciencia, Tecnología y Futuro, 4, 59-72.

[6] Schenk, P.M., Thomas-Hall, S.R., Stephens, E., Marx, U.C., Mussgnug, J.H., Posten, C., Kruse, O. and Hankamer, B. (2008) Second Generation Biofuels: High-Efficiency Microalgae for Biodiesel Production. BioEnergy Research, 1, 20-43.

https://doi.org/10.1007/s12155-008-9008-8

[7] Nigam, P.S. and Singh, A. (2010) Production of Liquid Biofuels from Renewable Resources. Progress in Energy and Combustion Science, 37, 52-68. https://doi.org/10.1016/j.pecs.2010.01.003

[8] Blanken, W., Postma, P.R., de Winter, L., Wijffels, R.H. and Janssen, M. (2016) Predicting Microalgae Growth. Algal Research, 14, 28-38.

[9] Bitog, J.P., Lee, I.-B., Lee, C.-G., Kim, K.-S., Hwang, H.-S., Hong, S.-W., Seo, I.-H., Kwon, K.-S. and Mustafa, E. (2011) Application of Computational Fluid Dynamics for Modelling and Designing Photo Bioreactors for Microalgae Production: A Review. Computers and Electronics in Agriculture, 76, 131-147. https://doi.org/10.1016/j.compag.2011.01.015

[10] Rengel, A. (2008) Promising Technologies for Biodiesel Production from Algae Growth Systems. Proceedings of the 8th European IFSA Symposium, Clermont-Ferrand, 6-10 July 2008, 683-692.

[11] Tredici, M.R. (2010) Photobiology of Microalgae Mass Cultures: Understanding the Tools for the Next Green Revolution. Biofuels, 1, 143-162.

[12] Kunjapur, A.M. and Eldridge, R.B. (2010) Photobioreactor Design for Commercial Biofuel Production from Microalgae. Industrial \& Engineering Chemistry Research, 49, 3516-3526. https://doi.org/10.1021/ie901459u

[13] Ugwu, C.U., Aoyagi, H. and Uchiyama, H. (2008) Photobioreactors for Mass Cultivation of Algae. Bioresource Technology, 99, 4021-4028. https://doi.org/10.1016/j.biortech.2007.01.046

[14] Deb, U.K., Chayantrakon, K. and Lenbury, Y. (2012) Comparison of Single-Phase and Two-Phase Flow Dynamics in the HLTP for Microalgae Culture. International Journal of Mathematics and Computers in Simulation, 6, 496-503.

[15] Pruvost, J., Pottier, L. and Legrand, J. (2006) Numerical Investigation of Hydrodynamic and Mixing Conditions in a Torus Photobioreactor. Chemical Engineering Science, 61, 44764489. https://doi.org/10.1016/j.ces.2006.02.027

[16] Molina, E., Fernandez, J., Acien, F.G. and Chisti, Y. (2001) Tubular Photobioreactor Design for Algal Cultures. Journal of Biotechnology, 92, 113-131. https://doi.org/10.1016/S0168-1656(01)00353-4

[17] Khanam, I.A. and Deb, U.K. (2016) Calculation of the Average Irradiance and the Microalgae Growth for a Year at CUET, Bangladesh. American Journal of Computational Mathematics, 6, 237-244. https://doi.org/10.4236/ajcm.2016.63024

[18] Deb, U.K., Kittisak, C., Lenbury, Y. and Benchawan, W. (2012) Numerical Simulation of 2-p Laminar Flow for $\mathrm{CO}_{2}$ and Microalgae Suspension in the HLTP. In: Latest Advances in Systems Science and Computational Intelligence, Wseas LLC, 53-58.

[19] Einstein, A. (1906) Eine neue Bestimmung der Moleküldimensionen. Annalen der Physik, 324, 289-306. https://doi.org/10.1002/andp.19063240204

[20] Hon-Nami, K. and Kunito, S. (1998) Microalgae Cultivation in a Tubular Bioreactor and Utilization of Their Cells. Chinese Journal of Oceanology and Limnology, 16, 75-83. https://doi.org/10.1007/BF02849084 
Submit or recommend next manuscript to SCIRP and we will provide best service for you:

Accepting pre-submission inquiries through Email, Facebook, LinkedIn, Twitter, etc. A wide selection of journals (inclusive of 9 subjects, more than 200 journals)

Providing 24-hour high-quality service

User-friendly online submission system

Fair and swift peer-review system

Efficient typesetting and proofreading procedure

Display of the result of downloads and visits, as well as the number of cited articles

Maximum dissemination of your research work

Submit your manuscript at: http://papersubmission.scirp.org/

Or contact ajcm@scirp.org 